\section{Corynebacterium macginleyi isolated from a corneal ulcer}

\author{
Kathryn L. Ruoff,' Christine M. Toutain- \\ Kidd, ${ }^{2}$ Muthiah Srinivasan, ${ }^{3}$ \\ Prajna Lalitha, ${ }^{3}$ Nisha R. Acharya, ${ }^{4,5}$ \\ Michael E. Zegans, ${ }^{2}$ \\ Joseph D. Schwartzman' \\ 'Department of Pathology, Dartmouth- \\ Hitchcock Medical Center, Lebanon, NH, \\ USA; ${ }^{2}$ Department of Surgery (section of \\ Ophthalmology), Dartmouth Medical \\ School, Hanover, NH, USA, and \\ Dartmouth-Hitchcock Medical Center, \\ Lebanon, NH, USA; ${ }^{3}$ Aravind Eye Care \\ System, India; ${ }^{4}$ F.I. Proctor Foundation, \\ University of California, San Francisco, \\ CA, USA; ${ }^{5}$ Department of \\ Ophthalmology, University of California, \\ San Francisco, CA, USA
}

\section{Abstract}

We report the isolation of Corynebacterium macginleyi from the corneal ulcer culture of a patient, later enrolled in the Steroids for Corneal Ulcer Trial (SCUT). To our knowledge this is the first published report from North America of the recovery of $C$. macginleyi from a serious ocular infection.

\section{Case Report}

An 84-year-old man with a previous history of Fuchs' endothelial dystrophy, Parkinson's syndrome, and bilateral cataract surgery presented to the Dartmouth-Hitchcock Medical Center ophthalmology clinic with a two-day history of decreased vision, eye pain, and tenderness in his right eye. He denied a history of trauma or contact lens wear. His Snellen visual acuity using his current glasses was $3 / 200$ in his right eye and he had an intra-ocular pressure of $19 \mathrm{mmHg}$. Slit lamp examination revealed a para-central $2.5 \mathrm{~mm}$ epithelial defect overlying a $3.6 \mathrm{~mm}$ corneal ulcer. There was minimal thinning of the corneal stroma and no hypopyon (see Figure 1). However, an endothelial plaque of white blood cells underlying the ulcer and +1 anterior chamber cells were present. The patient's eyelids were notable for blepharitis and meibomitis.

Corneal scrapings were obtained and inoculated onto T-soy agar containing 5\% sheep blood and chocolate agar (Remel, Lenexa, KS, USA). Growth at each inoculation point was noted on blood agar after incubation for 48 hours at $35^{\circ} \mathrm{C}$ in an atmosphere containing 3 $5 \% \mathrm{CO}_{2}$, but no growth occurred on chocolate agar. The small, slowly growing catalase-positive colonies were gray and non-hemolytic. Gram stains showed diphtheroidal gram-positive rods.

Dartmouth-Hitchcock Medical Center is a study site for the Steroids for Corneal Ulcer Trial (SCUT), which is a multicenter National Eye Institute (NEI) funded trial investigating the question of whether topical corticosteroids improve the outcome of bacterial keratitis when used in conjunction with topical antibiotics. Because this patient met the enrollment criteria for this study, participation in the study was offered to him and he elected to enroll. Consequently, he was treated according to the study protocol with topical $0.5 \%$ moxifloxacin hydrochloride applied every hour initially and the study medication, either topical $1 \%$ prednisolone phosphate or placebo, for three weeks. Patient enrollment in SCUT is ongoing and thus the nature of the treatment regimen that this patient received continues to remain masked to the clinical investigators. At the end of the three weeks of treatment, the patient's vision in his right eye had improved to $20 / 320$, and by three months it was back to the pre-ulcer vision at $20 / 100$.

The catalase-positive gram-positive rod isolated from the patient's culture was identified as $C$. macginleyi by the API Coryne identification system (BioMerieux, Durham, NC, USA). The organism's profile number, 1100305, was noted previously ${ }^{1,2}$ as one of the profiles exhibited by the $C$. macginleyi strains isolated from ocular specimens. Growth of the organism on blood but not chocolate agar suggested a lipophilic nature as previously described for $C$. macginleyi. ${ }^{1}$ The organism's lipophilic behavior was demonstrated with Tween 80 (Sigma, St. Louis, M0, USA) supplemented media. Growth was stimulated (colony size approximately $2 \mathrm{~mm}$ in diameter after 48 hours of incubation) on sheep blood agar plates that had been supplemented with $0.1 \mathrm{~mL}$ of sterile $10 \%$ Tween 80 compared to growth on non-supplemented blood agar (colony size approximately $0.5 \mathrm{~mm}$ after 48 hours of incubation). Growth also occurred on Tween-supplemented but not on unsupplemented chocolate agar.

The isolate was identified further by $16 \mathrm{~S}$ rRNA gene sequencing. Nucleic acid was prepared with PrepMan Ultra reagent (Applied Biosystems, Foster City, CA, USA) and PCR was performed with a MicroSeq 500 kit (Applied Biosystems) according to the manufacturer's instructions. ExoSAP-IT (USB Co., Cleveland, OH, USA) was used to purify the PCR product, which was sequenced at the Dartmouth College Molecular Biology and Proteomic Core Facility. The National Center for Biotechnology Information (NCBI) GenBank BLAST program was used to identify
Correspondence: Kathryn L. Ruoff, Department of Pathology (Microbiology), Dartmouth Hitchcock Medical Center, One Medical Center Drive, Lebanon, NH 03756, USA.

E-mail: kathryn.l.ruoff@Hitchcock.org

Key words: corynebacterium macginleyi, cornea, ulcer.

Contributions: KLR and JDS were involved in the identification of the $C$. macginleyi isolate; KLR prepared the manuscript; CMTK and MEZ were involved in the patient's follow-up and treatment, as well as the preparation of the manuscript; MS, $\mathrm{PL}$, and NRA were involved in the design of the study and overall management of the patient. All authors have read and approved the final version of the manuscript.

Acknowledgements: the SCUT study (CMTK, MS PL, NRA, and MEZ) is supported by the National Eye Institute (NEI, U10 EY015114).

Conflict of interest: the authors report no conflicts of interest.

Received for publication: 11 December 2009

Revision received: 8 February 2010.

Accepted for publication: 8 February 2010.

This work is licensed under a Creative Commons Attribution 3.0 License (by-nc 3.0).

(C) Copyright K.L. Ruoff et al., 2010

Licensee PAGEPress, Italy

Infectious Disease Reports 2010; 2:e3 doi:10.4081/idr.2010.e3

homologous sequences. The sequence of our isolate displayed $99 \%$ similarity to sequences from a number of previously described $C$. macginleyi isolates (accession numbers AB359405, AB359393, AJ439345, X80499).

The patient's isolate displayed minimum inhibitory concentrations for penicillin, ciprofloxacin, tobramycin, and vancomycin of $0.016,0.032,0.064$, and $0.5 \mu \mathrm{g} / \mathrm{mL}$, respectively, when tested by the Etest method ( $A B$ Biodisk, Solna, Sweden) on sheep blood-supplemented Mueller Hinton agar (Remel). These observations were similar to previously reported susceptibilities of the majority of $\mathrm{C}$. macginleyi strains isolated from ocular specimens, ${ }^{1,3,4}$ but a recent report ${ }^{5}$ noted fluoroquinolone and sulbenicillin resistance in two ocular strains. Isolates from other body sites ${ }^{4,6,7}$ have shown more resistant phenotypes.

\section{Discussion}

Few reports of $C$. macginleyi isolated from clinical isolates have appeared since this species was proposed by Riegel and colleagues in $1995 .^{8}$ The majority of isolates described 


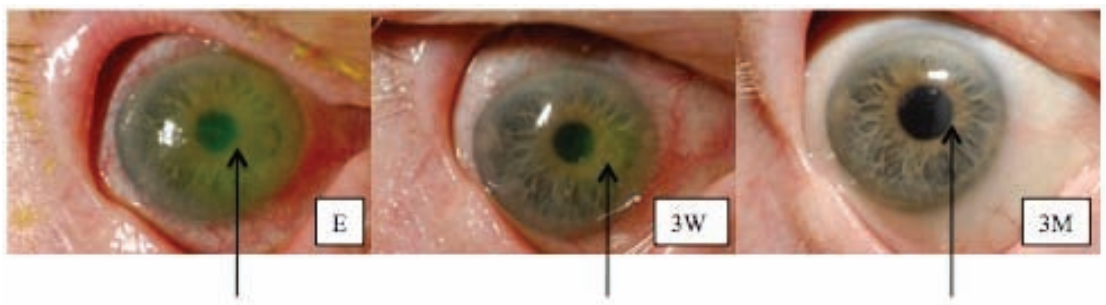

Figure 1. Photographs of the infected eye taken on the first day of treatment $(E)$, after 3 weeks $(3 \mathrm{~W})$, and after 3 months of follow-up $(3 \mathrm{M})$. The arrow indicates the site of the ulcer $(E$ and $3 \mathrm{~W})$ and the scar $(3 \mathrm{M})$. Please note that the yellow-green coloration of the ulcer in photograph $\mathrm{E}$ is due to fluorescein drops used during examination of the patient's cornea. Staining with fluorescein indicates disruption of the corneal epithelium. Staining with fluorescein indicates disruption of the corneal epithelium.

have been recovered from conjunctival swab specimens, ${ }^{1-3,9}$ and $C$. macginleyi is thought to be a component of normal conjunctival flora that may function as an opportunistic pathogen in conjunctivitis as well as other ocular infections. $C$. macginleyi has rarely been associated with more serious ocular infections such as keratitis, ${ }^{5,10}$ and endophthalmitis. ${ }^{11}$ Three case reports described $C$. macginleyi as an agent of infection in non-ocular sites, including the urine of a patient with a permanent bladder catheter, ${ }^{4}$ an infected intravenous catheter, ${ }^{6}$ and a case of septicemia. ${ }^{7}$ The association of $C$. macginleyi infections with the presence of prosthetic abiotic materials as described above, suggests that bacterial biofilm formations on these surfaces may play a role in its pathogenesis. However, the presence of a bacterial biofilm has been documented only in the suture-related keratitis case described by Suzuki and colleagues. ${ }^{5}$

Corynebacteria, for the most part, are susceptible to a wide array of antibiotics such as penicillins, macrolides, rifampicin, gentamicin, and fluoroquinolones, ${ }^{1,12}$ although one recent report from Japan showed that 11 out of 16 C. macginleyi ophthalmic isolates were resistant to fluoroquinolones. ${ }^{9}$ It is difficult to know why no $C$. macginleyi case has ever been reported in North America. Identification techniques have evolved tremendously in recent years; the API Coryne system appears to be accurate for identification of this organism, ${ }^{1}$ which may have been characterized only to the genus level in the past. Slow growth on blood agar of lipophilic diphtheroidal organisms isolated from ocular cultures should alert microbiologists to the possible presence of $C$. macginleyi. This bacterial species seems to be encountered infrequently. For instance, in a report studying the normal bacterial flora of the conjunctiva, only four isolates out of the 25 corynebacteria identified were $C$. macginley $i^{13}$ To our knowledge, this is also the first time that this bacterium has been identified in an eye isolate at Dartmouth Hitchcock Medical Center. Finally, it is possible that infections caused by $C$. macginleyi are less noticeable since they are frequently successfully treated with a standard regimen of antibiotics.

Our patient had at least two distinct risk factors for bacterial keratitis. He had Fuchs' endothelial dystrophy, a dysfunction of the corneal endothelium that can lead to corneal edema, and compromise of the corneal epithelial barrier. ${ }^{14}$ Gaps in the integrity of the corneal epithelial layer caused by excessive contact lens wear, trauma, or diseases compromising the ocular surface such as Fuchs', are well known to predispose patients to bacterial corneal infection..$^{15}$ Furthermore, Parkinson's disease has been reported to alter tear function in most patients, further compromising the ocular surface. ${ }^{16}$ Thus, our patient had an impaired ocular surface likely rendering his cornea particularly permissive to infection with what is normally a commensal organism, C. macginleyi. Because of the relatively small size of the ulcer, its location, and the good response to treatment, the patient's vision in the right eye returned to its pre-keratitis level (Figure 1).

All reports of isolation of $C$. macginleyi published to date have originated in Europe, Australia, or Japan. We document a case of corneal ulcer yielding $C$. macginleyi as the sole isolate. To our knowledge the case reported here represents the first published account of clinical isolation of this organism in North America.

\section{References}

1. Funke G, Pagano-Niederer M, Bernauer W. Corynebacterium macginleyi has to date been isolated exclusively from conjunctival swabs. J Clin Microbiol 1998;36:3670-3.

2. Joussen AM, Funke G, Joussen F, et al. Corynebacterium macginleyi: a conjunctiva specific pathogen. Br J Ophthalmol 2000;84:1420-2.

3. Giammanco GM, Di Marco V, Priolo I, et al. Corynebacterium macginleyi isolation from conjunctival swab in Italy. Diag
Microbio Infect Dis 2002;44:205-7.

4. Villanueva JL, Dominguez A, Rios MJ, et al. Corynebacterium macginleyi isolated from urine in a patient with a permanent bladder catheter. Scan J Infect Dis 2002; 34:699-700.

5. Suzuki T, lihara H, Uno T, et al. Suturerelated keratitis caused by Corynebacterium macginleyi. J Clin Microbiol 2007; 45:3833-6.

6. Dobler GBI. Highly resistant Corynebacterium macginleyi as cause of intravenous catheter-related infection. Eur J Clin Microbiol Infect Dis 2003;22:72-3.

7. Villamil-Cajoto I, Rodriguez-Otero L, Villacian-Vicedo M.J. Septicemia caused by Corynebacterium macginleyi: a rare form of extraocular infection. Int $\mathrm{J}$ Infec Dis 2008; 12:333-5.

8. Riegel P, Ruimey $\mathrm{R}$, deBriel $\mathrm{D}$, et al. Genomic diversity and phylogenetic relationships among lipid-requiring diphtheroids from humans and characterization of Corynebacterium macginleyi sp. Int J Syst Bacteriol 1995;45:128-33.

9. Eguchi H, Kuwahara T, Miyamoto T, et al. High-level fluoroquinolone resistance in ophthalmic clinical isolates belonging to the species Corynebacterium macginleyi. J Clin Microbiol 2008;46:527-32.

10. Ly CN, Pham JN, Badenoch PR, et al. Bacteria commonly isolated from keratitis specimens retain antibiotic susceptibility to fluoroquinolones and gentamicin plus cephalothin. Clin Exp Ophthalmol 2006; 34:44-50.

11. Ferrer C, Ruiz-Moreno J, Rodriguez A, et al. Postoperative Corynebacterium macginleyi endophthalmitis. J Cataract Refract Surg 2004;30:2441-4.

12. Somodevilla A, Domingo D, Martinez MC, et al. Corynebacterium macginleyi: susceptibility to 7 usual ophthalmic antibiotics. 19th European Congress of Clinical Microbiology and Infectious Diseases Helsinki, Finland; 2009.

13. von Graevenitz A, Schumacher U, Bernauer W. The Corynebacterial Flora of the Normal Human Conjunctiva Is Lipophilic. Curr Microbiol 2001;42:372-4.

14. Borboli S, Colby K. Mechanisms of disease: Fuchs' endothelial dystrophy. Ophthalmol Clin North Am 2002;15:17-25.

15. Keay L, Edwards J, Naduvilath T, et al. Microbial keratitis: predisposing factors and morbidity. Ophthalmology 2000;113: 109-16.

16. Tamer C, Melek I, Duman T, et al. Tear film tests in Parkinson's disease patients. Ophthalmology 2005;112:1795e1-e8. 